\title{
Symbol Rate Estimation based on Wavelet Transform and Cyclic Spectrum
}

\author{
Danyang $\mathrm{Li}^{\mathrm{a}}$, Xiaojun $\mathrm{Hao}^{\mathrm{a}}$, and Zhaoyue Zhang ${ }^{\mathrm{b}}$,* \\ ${ }^{a}$ State Key Laboratory of Complex Electromagnetic Environment Effects on Electronics and Information System (CEMEE), Luoyang, 471003, China \\ ${ }^{b}$ College of Air Traffic Management, Civil Aviation University of China, Tianjin, 300300, China
}

\begin{abstract}
Aiming at the digital modulation signals such as MASK, MPSK, and MQAM in a Gaussian white noise environment, this paper theoretically analyzes the reason and rule of the appearance of the spectrum of wavelet transform and the symbol rate spectrum line of cyclic spectrum. The simulation results show that the method based on wavelet transform is better than that based on cyclic spectrum, and the SNR threshold of the first method is reduced by $8 \mathrm{~dB}$ compared with the latter.
\end{abstract}

Keywords: cyclic spectrum; digital modulated signal; root raised cosine filter; wavelet transform

(Submitted on April 12, 2019; Revised on May 29, 2019; Accepted on June 19, 2019)

(C) 2019 Totem Publisher, Inc. All rights reserved.

\section{Introduction}

The symbol rate is the most commonly used parameter in digital communication. Nowadays, with more and more frequent information communications, signal interception, monitoring, and interference are indispensable in both life and military affairs. For civilian use, managers need to listen for illegal communications. For the military, electronic countermeasures require intercepting enemy intelligence. In the above scenario, an important step is to accurately estimate the signal symbol rate, so as to lay a solid foundation for other steps.

For the estimation of symbol rate, Han et al. proposed a new method of combination of bandwidth estimation, wavelet transform, and statistical analysis, which has high accuracy and robustness [1]. Zhou also put forward a kind of transform of the signal, and using this transform, an accurate spectral line associated with the symbol rate was obtained [2]. A method based on cyclic field energy profile was studied in 2014 by Phukan [3]. In the same year, a method based on analysis of the autocorrelation frequency response was studied by Ahmet, because he found that near the symbol rate, there was a large oscillation. This method had a certain complexity [4]. In 2015, Majhi proposed a blind bit rate estimation method for linear modulation signals and estimated the bit rate of OQPSK signals without knowing the modulation scheme [5]. Yang et al. analyzed the envelope spectrum of the signal and found that the symbol rate can be estimated by its pulse line [6]. The method using cyclostationary characteristics in the wavelet domain was proposed by Kumar in 2016 [7]. In 2017-2018, more new methods were proposed. For the estimation of the symbol rate of the BPSK signal, Pei et al. chose the method based on Hankel-singular value decomposition (SVD) [8]. Kuchumov et al. estimated the number element rate of MFSK and MPK signals through subcarrier decomposition of the signal [9]. In 2018, Liu first calculated the spectrum of teager energy operator (TEO) for MPSK signal, and then, using the position of the spectrum line of TEO, the symbol rate of signal was estimated [10].

In this paper, we use the method of wavelet transform and cyclic spectrum to obtain the estimation results of the symbol rate of nine kinds of digital modulation signals.

\footnotetext{
* Corresponding author.

E-mail address: zyzhang@ cauc.edu.cn
} 


\section{Method based on Wavelet Transform and Cyclic Spectrum}

\subsection{Symbol Rate Estimation based on Cyclic Spectrum}

A cyclostationary signal is a kind of signal whose mathematical expectation and auto-correlation function change periodically [11]. It is different from the periodic signal and stationary signal; these two kinds of signals are only a special case of it. Suppose the expectation of the cyclostationary signal $x(t)$ is $m_{x}(t)$, then the periodicity of $m_{x}(t)$ should be guaranteed by $x(t)$ in the first place.

$$
m_{x}(t+k T)=m_{x}(t)
$$

If the autocorrelation function of $x(t)$ is $R_{x}(t, \tau)$, then $R_{x}(t, \tau)$ also needs to be periodic.

$$
R_{x}(t+k T, \tau)=R_{x}(t, \tau)
$$

$R_{x}(t, \tau)$ can be calculated from the following formula.

$$
R_{x}(t, \tau)=\sum_{\alpha} R_{x}^{\alpha}(\tau) e^{j 2 \pi \alpha t}
$$

Let $\alpha=n / T$ be the cycle frequency, then the time average form of $R_{x}(t, \tau)$ can be expressed by

$$
R_{x}^{T}(\alpha, \tau)=\lim _{T \rightarrow \infty} \frac{1}{T} \int_{-T / 2}^{T / 2} R_{x}(t, \tau) \exp (-j 2 \pi \alpha t) \mathrm{d} t
$$

The signal in the above formula does not have ergodicity; if it has ergodicity, its calculation method can be simplified as

$$
R_{x}^{T}(\alpha, \tau)=\lim _{T \rightarrow \infty} \frac{1}{T} \int_{-T / 2}^{T / 2} x\left(t-\frac{\tau}{2}\right) x^{*}\left(t+\frac{\tau}{2}\right) \exp (-j 2 \pi \alpha t) \mathrm{d} t
$$

For digital signals, their symbol rates are closely related to the position of the characteristic line in the cyclic spectrum. Usually, there is an integer multiple relationship between the two. When the cyclic spectral correlation method is used for parameter estimation, the cyclic frequency resolution $\Delta \alpha$ and the frequency resolution $\Delta f$ have great influence on the precision of symbol rate estimation. In this paper, when calculating the cyclic spectrum of the signal, we set the resolution a bit coarser in order to decrease the execution time of the algorithm and guarantee a certain estimation effect. We set the cyclic resolution $\Delta \alpha$ and frequency resolution $\Delta f$ as $f_{s} / N^{\prime}$, where $N^{\prime}=N / 8, N$ is the data length, and $f_{s}$ is the sampling rate.

We take 2PSK as an example to introduce the method of estimating symbol rate by using the cyclic spectrum. Figure 1 is the cyclic spectrum of 2PSK. The simulation environment is an ideal environment without noise. The parameters are: carrier frequency $8 \mathrm{KHz}$, symbol rate $2000 \mathrm{Sps}$, sampling rate $32 \mathrm{KHz}$, and signal length 2048 points.

The arg_ $\alpha[\bullet]$ operation represents the extraction of cyclic frequency axis coordinates of $\bullet$, and the arg_f $f \bullet]^{-}$ operation represents the extraction of frequency axis coordinates of $\bullet . \Delta \alpha$ is the cyclic frequency resolution, and $\Delta f$ is the frequency resolution.

The symbol rate can be estimated by using the cyclic spectrum in the following ways.

First, take $f$ axis coordinates $f_{\max }$ of the partial maximum value of $\left.S_{x}^{0}(f)\right|_{f>0}$, and then $f_{\max }$ can be expressed as

$$
f_{\max }=\arg f\left[\max \left(\left.S_{x}^{0}(f)\right|_{f>0}\right)\right]
$$




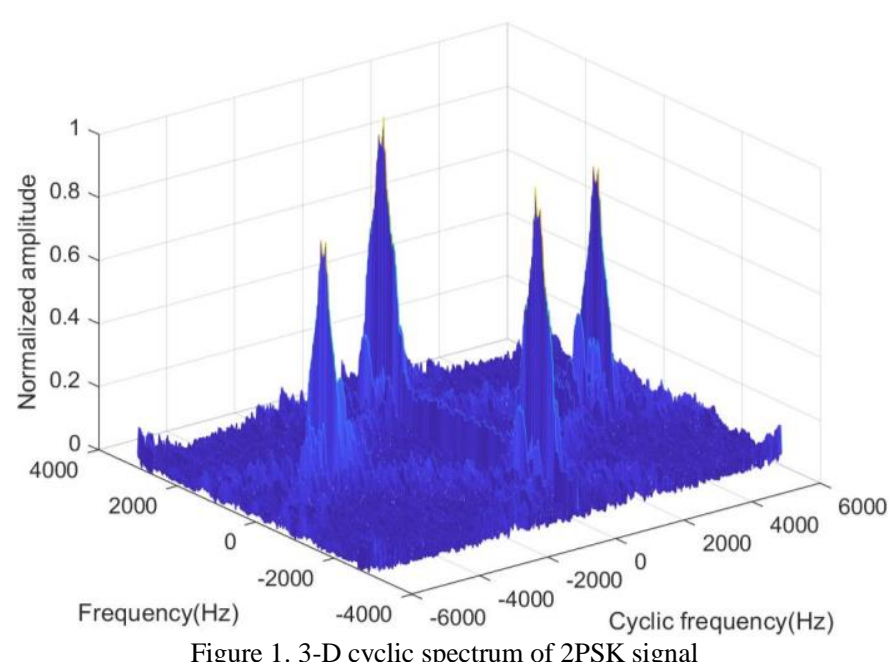

The section of circular frequency $\alpha=0$ is shown in Figure 2 .

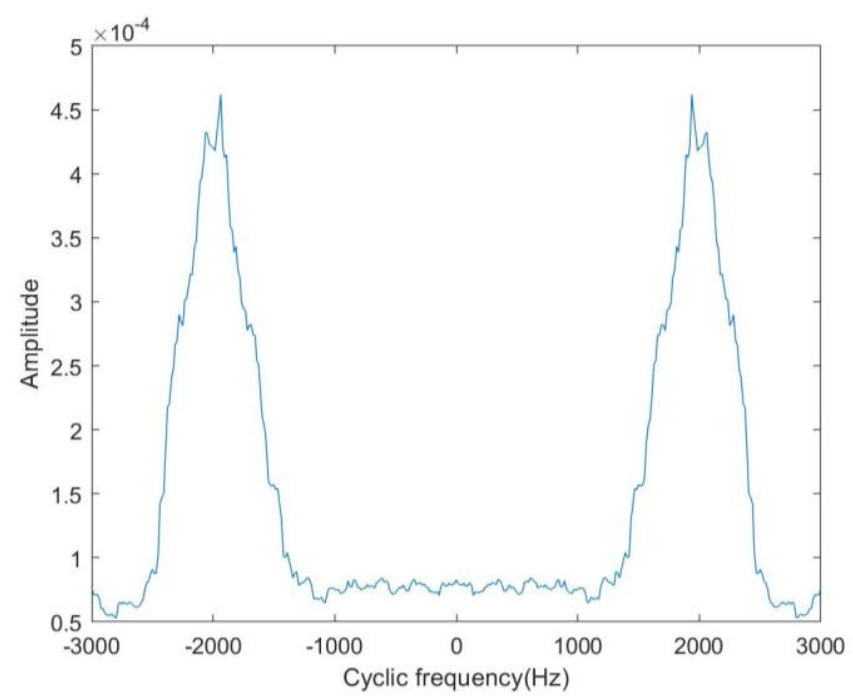

Figure 2. Section of the cycle frequency $\alpha=0$

According to this section, the maximum value of the right half axis is found, and its corresponding coordinate is $f_{\max }$.

Extract the circular frequency coordinates of the maximum value of plane $\left.S_{x}^{\alpha}\left(f_{\max }\right)\right|_{\alpha>0}:$ it is the estimation of the sign rate.

$$
\hat{f}_{d}=\arg \_\alpha\left[\max \left(\left.S_{x}^{\alpha}\left(f_{\max }\right)\right|_{\alpha>0}\right)\right] \times \Delta \alpha
$$

The section of frequency $f=f_{\max }$ is shown in Figure 3. According to this section, we can get the coordinate corresponding to the maximum point of the right half axis (excluding the zero point) and estimate the value of the symbol rate by calculating according to the above formula. We use the same method for other digital modulation signals and therefore do not elaborate on it here.

\subsection{Symbol Rate Estimation based on Wavelet Transform}

Continuous wavelet transform is used to expand the function $s(t)$ under the wavelet basis. Define $b$ as the displacement factor and $a$ as the scale factor, and then its coefficient is $W T_{s}(b, a)$ [12]. It can be written as 


$$
W T_{s}(b, a)=\frac{1}{\sqrt{a}} \int_{-\infty}^{+\infty} s(t) g^{*}\left(\frac{t-b}{a}\right) \mathrm{d} t=\left\langle s(t), g_{(b, a)}(t)\right\rangle
$$

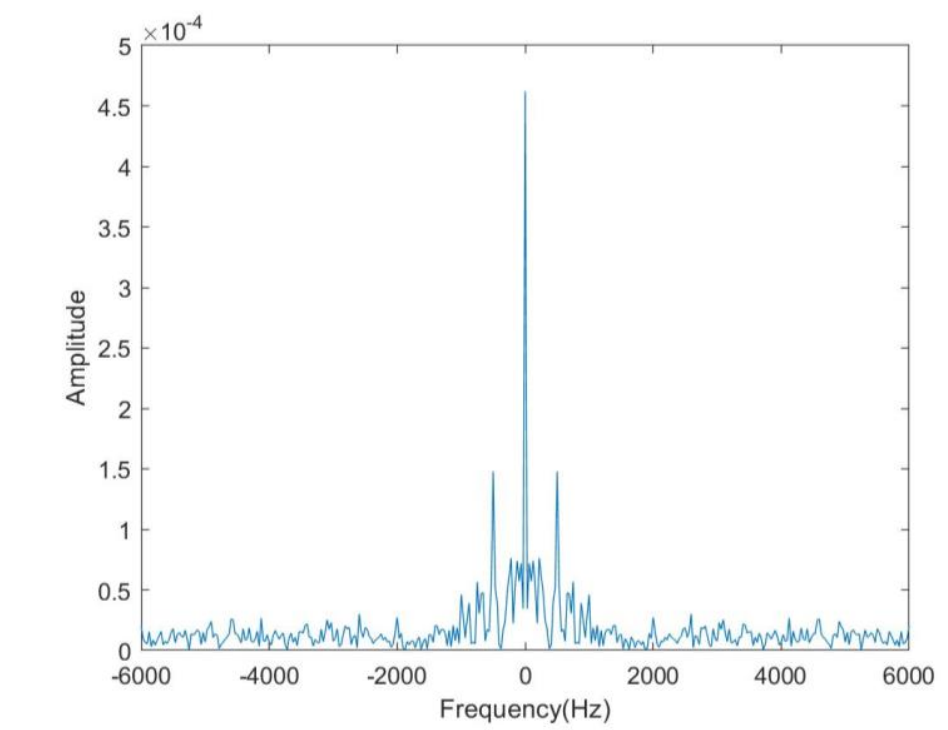

Figure 3. Section of frequency $f=f_{\max }$

The wavelet basis function is $g(t) \in L^{2}(R)$, and $L^{2}(R)$ is any space. Suppose the Fourier transform is $G(\omega)$, and then $g(t)$ has to satisfy

$$
W_{\psi}=\int_{-\infty}^{+\infty} \frac{|G(w)|}{|w|} \mathrm{d} w<\infty
$$

Through translation and extension of the wavelet basis function $g(t)$, the wavelet function family $g_{(b, a)}(t)$ is

$$
g_{(b a}(t) z^{-\frac{1}{2}} g\left(\frac{t-b}{a}\right) \quad b \in R \quad a,>
$$

For the digital modulation signal, the magnitude sequence of wavelet transform will also have a significant difference in the coding changing, that is, there will be equispaced peaks in the frequency domain. If $N_{s}$ is the number of Fourier transform points and $f_{s}$ is the sampling frequency, then, using the peak distance $d$, the estimated symbol rate $R_{s}$ can be calculated according to

$$
R_{s}=\frac{d}{N_{s}} \cdot f_{s}
$$

Where $R_{s}$ and $d$ are linear because $N_{s}$ and $f_{s}$ are fixed values.

Take the 2PSK signal as an example to introduce the method based on wavelet transform. Figure 4 is the spectrum diagram of the wavelet transform after downing converse signal to the base band. The wavelet basis function used by the wavelet transform is the Haar wavelet, and the parameters are: symbol rate 2000Sps, carrier frequency $8 \mathrm{KHz}$, sampling frequency $32 \mathrm{KHz}$, and signal length 2048 points.

As can be seen from Figure 4, the magnitude of the symbol rate can be obtained through the peak distance, because after the wavelet transform, the symbol rate of the 2PSK base band signal is equal to the peak distance of its spectrum. We use the same method for other digitally modulated signals and therefore do not elaborate on it here. 


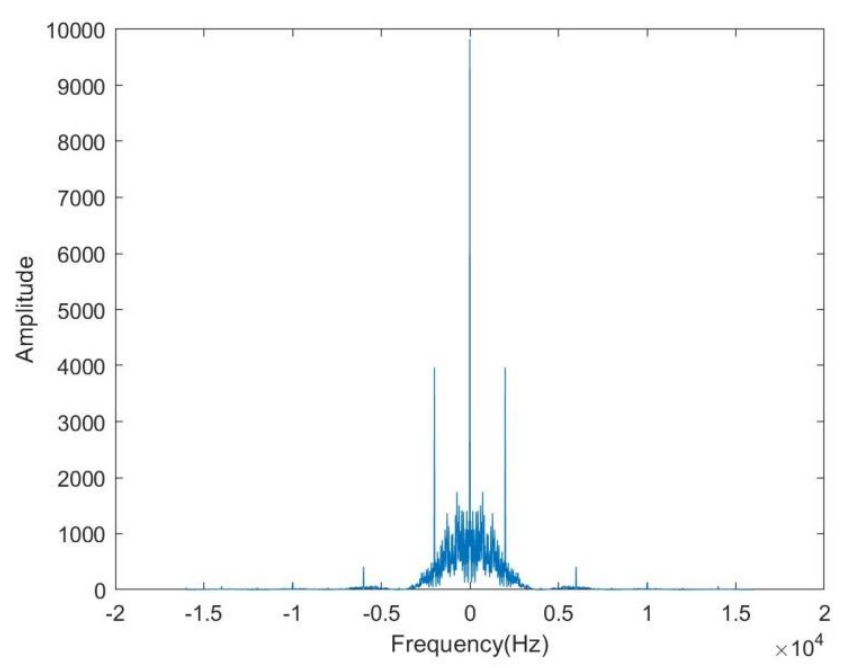

Figure 4. Spectrum of wavelet transform of 2PSK signal

\section{Simulation Analysis}

First, we take 2PSK as an example to carry out a comparative simulation experiment between the wavelet transform method and the cyclic spectrum correlation method. The results are estimated using the normalized mean square error (NMSE) as a benchmark. The parameters are as follows: symbol rate $2000 \mathrm{Sps}$, carrier frequency $4 \mathrm{KHz}$, sampling rate $64 \mathrm{KHz}$, and at each SNR, we perform 100 Monte Carlo experiments. Figure 5 shows the comparison of the two methods from -10db to $10 \mathrm{~dB}$ using 128 symbols.

As can be seen from Figure 5, the wavelet transform method always performs better than the cyclic spectral correlation method under the same signal-to-noise ratio. However, in the case of different SNR values, with an increase in the SNR, the value of the NMSE using the cyclic spectral correlation method shows a declining trend, that is, its estimation effect shows a better trend with the growth of SNR. Moreover, the NMSE tends to be stable when the SNR is higher than 3dB; it can stay below 0.01, and in the best case, it can reach 0.001. The NMSE of the wavelet transform method also shows a declining trend as the signal-to-noise ratio increases. It tends to be stable above $-5 \mathrm{~dB}$, which can basically remain at 0 . The above phenomenon is due to the good anti-noise performance of wavelet transform, so it can also have a good estimation effect at a low SNR and is always better than the effect of the cyclic spectral correlation method.

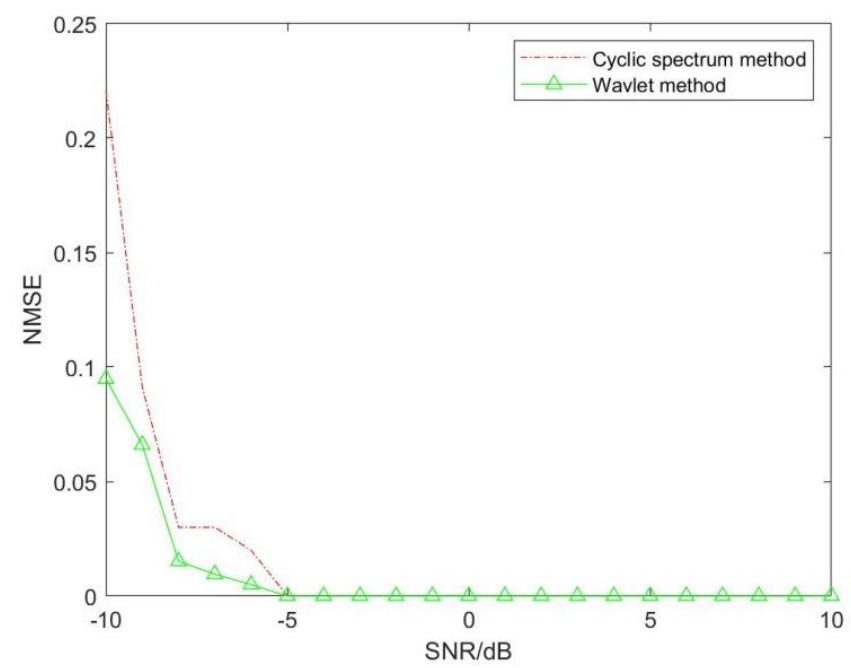

Figure 5. Simulation curves of two symbol rate estimation methods at different SNR values

Next, we carry out a comparative experiment for the two methods under different symbol numbers. Since the cyclic spectral correlation method can only estimate the signal whose length is an integer exponent times of 2, the variation range of the symbol number is $2^{4}$ to $2^{9}$, and the signal-to-noise ratio is $10 \mathrm{~dB}$. The other parameters are the same as those in the above experiments, and the simulation results are expressed in Figure 6. 
As shown in Figure 6, the wavelet transform method always performs better than the cyclic spectral correlation method under the same number of symbols. Under different symbol numbers, the estimation effect of the two methods becomes better as the symbol number increases.

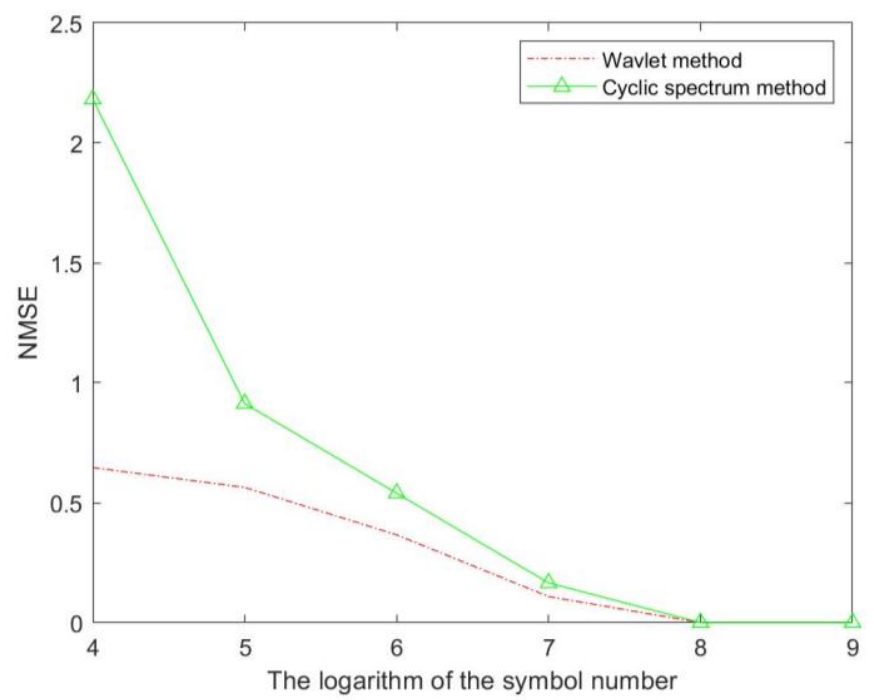

Figure 6. Simulation curves of two symbol rate estimation methods with different symbol numbers

Then, we carry out simulation experiments including the 11 kinds of digital signals mentioned in the second chapter at different SNR values. The parameters are the same as the first group of experiments, and the SNR ranges from -10dB to $10 \mathrm{~dB}$. The simulation results of the two methods are displayed in Figures 7 and 8.

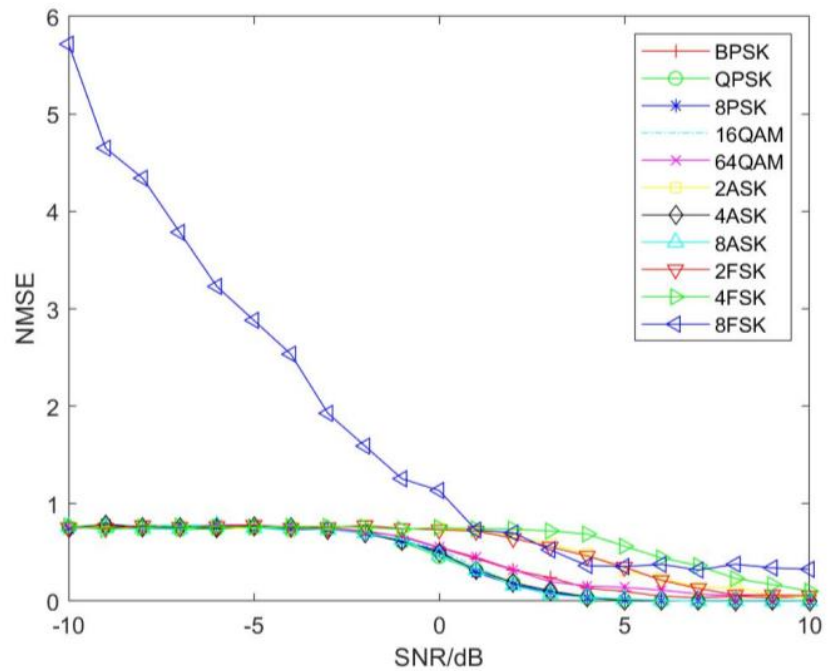

Figure 7. Simulation curves of the cyclic spectral correlation method for 11 signals at different SNR values

As can be seen from Figures 7 and 8, for the cyclic spectral correlation method, the estimation effect of all signals becomes better as the signal-to-noise ratio increases. In addition, when the SNR is above 6dB, it tends to be stable, and the NMSE can be kept below 0.5. Except for the three FSK signals, all of them can be stabilized below 3dB, with the NMSE below 0.01. This shows that the estimation effect of the FSK signal is not as good as that of other signals. For the wavelet transform method, except for the 8FSK signal, all other signals can be stabilized above -6dB, and the NMSE can be maintained below 0.6. Except for the three kinds of FSK signals and 2ASK signal, the NMSE of other signals can approach 0 when the SNR is $-6 \mathrm{~dB}$. Therefore, regardless of which method is used, the symbol rate estimation result of the FSK signal is generally not as good as that of other signals, because FSK signal spectrum characteristics are more complicated. The symbol rate should be estimated according to the spectral characteristics, so the estimation effect of such signals is not as good as that of other signals with simple spectral features. 


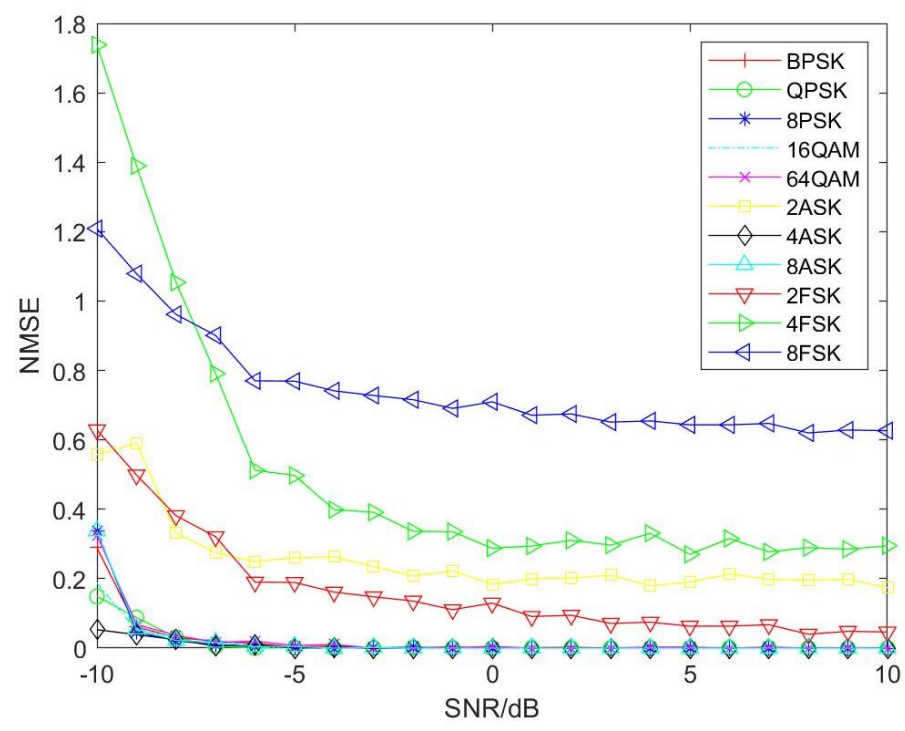

Figure 8. Simulation curves of the wavelet transform method for 11 signals at different SNR values

\section{Conclusions}

In this paper, to estimate the symbol rate, the method based on cyclic spectrum and wavelet transform is studied, where the NMSE of the cyclic spectrum method can reach below 0.01 at SNR > -3dB, while the NMSE value of the wavelet transform method can reach below 0.001 at $\mathrm{SNR}>-6 \mathrm{~dB}$. This shows that the estimation effect of the wavelet transform method is better than that of the cyclic spectrum method.

\section{Acknowledgments}

The authors would like to thank the State Key Laboratory of Complex Electromagnetic Environment Effects on Electronics and Information System Director Fund (CEMEE2019Z0105B).

\section{References}

1. Z. Su, J. Xu, and M. Han, "Symbol Rate Estimation of MPSK Short Signals based on Wavelet Transform," Journal of Electronic Measurement and Instrument, Vol. 27, No. 2, pp. 140-144, February 2013

2. P. Zhou and C. S. Li, "A Novel Symbol Rate Estimation Algorithm for Phase Modulating Signals in Wireless Communications," Applied Mechanics and Materials, Vol. 443, No. 1, pp. 392-396, October 2013

3. G. J. Phukan and P. K. Bora, "An Algorithm for Blind Symbol Rate Estimation using Second Order Cyclostationarity," in Proceedings of International Conference on Signal Processing \& Communications, pp. 1-6, Bangalore, India, December 2014

4. A. Guner and I. Kaya, "Periodic Variation Method for Blind Symbol Rate Estimation," in Proceedings of IEEE 22nd International Symposium on Personal Indoor and Mobile Radio Communication, pp. 147-151, Toronto, Canada, January 2012

5. S. Majhi and T. S. Ho, "Blind Symbol-Rate Estimation and Test Bed Implementation of Linearly Modulated Signals," IEEE Transactions on Vehicular Technology, Vol. 64, No. 3, pp. 954-963, March 2015

6. W. Yang and X. Yang, "Research on Symbol Rate Estimation based on the Generalized Square Envelope Spectrum," in Proceedings of IEEE International Conference on Communication Problem-Solving, pp. 391-394, Guilin, China, April 2016

7. S. Kumar, V. A. Bohara, and S. J. Darak, "Blind Symbol Rate Estimation by Exploiting Cyclostationary Features in Wavelet Domain," in Proceedings of International Conference on Advances in Computing, pp. 1757-1763, Jaipur, India, November 2016

8. Z. Y. Qiu, P. Wang, J. Zhun, and B. Tang, "Estimation of Both Nyquist Zone Index and Code Rate for BPSK Radar Signal Intercepted by Nyquist Folding Receiver," IET Radar, Sonar \& Navigation, Vol. 11, No. 11, pp. 1652-1663, November 2017

9. A. A. Kuchumov, V. I. Lipatkin, and E. M. Lobov, "Advanced Algorithms for Automatic Symbol Rate Estimation of M-FSK and M-PSK Signals," Systems of Signal Synchronization, Generating and Processing in Telecommunications, pp. 1-4, Kazan, Russia, July 2017

10. M. Q. Liu, J. Zhang, and B. Li, "Symbol Rates Estimation of Time-Frequency Overlapped MPSK Signals for Underlay Cognitive Radio Network," IEEE Access, Vol. 6, pp. 16216-16223, March 2018

11. F. He, Y. Yin, L. Zhou, and X. Z. Xu, "Principal Component Analysis of Cyclic Spectrum Features in Automatic Modulation Recognition," in Proceedings of Military Communications Conference, pp. 1737-1742, San Jose, USA, January 2011

12. K. Hassan, I. Dayoub, W. Hamouda, and M. Berbineau, "Automatic Modulation Recognition using Wavelet Rransform and Neural Network," in Proceedings of International Conference on Intelligent Transport Systems Telecommunications, pp. 234-238, Lille, France, January 2010 